\title{
Universal and Nonuniversal Dynamical Conductivity in Small Metallic Grains: An Ambivalent Role of T-Invariance at Finite Frequency
}

\author{
Nobuhiko Taniguchi \\ Institute of Physics, University of Tsukuba, Tennodai Tsukuba 305-8571, Japan \\ Correspondence should be addressed to Nobuhiko Taniguchi, taniguch@cm.ph.tsukuba.ac.jp
}

Received 1 September 2009; Revised 12 May 2010; Accepted 15 May 2010

Academic Editor: Zhidong Bai

Copyright (C) 2010 Nobuhiko Taniguchi. This is an open access article distributed under the Creative Commons Attribution License, which permits unrestricted use, distribution, and reproduction in any medium, provided the original work is properly cited.

\begin{abstract}
The idea of random matrix theory is applicable not only to the level statistics but also to various physical observables. Taking the dynamical conductivity in isolated quantum dots with diffusive dynamics, we investigate analytically intertwining effects of the time-reversal invariance, level repulsion and quantum interference. We clarify an ambivalent role of the time-reversal invariance at finite frequency by a new invariant analysis respecting the symmetry of the effective field theory. A subtlety of the operator insertion, and the fast-slow mode separation within the effective field description is pointed out.
\end{abstract}

\section{Introduction}

Since the dawn of mesoscopic physics, dynamical properties of small metallic grains have been attracting much attention both in experiments and in theories; they have been recognized as very useful physical realizations of the random matrix theory, because energy levels of such systems are known to exhibit strong repulsion as in random matrices. From a theoretical point of view, dynamical responses are much more complicated to investigate than energy level statistics. It is because the former requires an additional knowledge of electron wavefunctions or matrix elements in addition to energy spectra.

In a pioneering paper by Gor'kov and Eliashberg (GE) [1], a phenomenological theory of dynamical responses was built based on the two statistical hypothesis: (i) independent fluctuations between matrix elements and energy levels (to single out the effect of the level repulsion), and (ii) the DOS correlator approximated by the Wigner-Dyson level correlator 
[2]. Although the original work was found to be insufficient in taking account of interaction effect $[3,4]$, the statistical approach has been widely accepted as a building block for subsequent studies $[5,6]$.

Although statistical hypothesis seems plausible, its microscopic justification is hard and often requires a sophisticated method. The parameter controlling the validity is the dimensionless conductance $g$ (for diffusive systems, $g=D / L^{2} \Delta$ where $D$ is the diffusion coefficient in a linear size $L$ and $\Delta$ is the mean level spacing). To our knowledge, the only adequate tool to employ is the supermatrix nonlinear sigma (NL- $\sigma$ ) approach [7], which proved the hypothesis (ii) in diffusive quantum dots with $g \approx \infty$. Justifying the "decoupling" hypothesis (i) came rather late $[8,9]$. However, such decoupling scheme may be oversimplified. Indeed, a new quantum effect sensitive to the time-reversal invariance (Tinvariance) was observed in the limit of $g \approx \infty$ as a flux-dependent polarizability at $\omega \lesssim \Delta$ $[10,11]$. The effect has been unnoticed in the treatments based on the statistical hypothesis. This new effect should also affect the dynamical magnetto-conductivity, whose magnetic dependence can be either positive or negative by the frequency observed [12,13].

In this paper, utilizing the supermatrix formulation, we present a full analysis of the quantum effect on the dynamical conductivity $\sigma(\omega)$ in isolated small metallic grains. Unlike previous similar attempts, our approach encompasses all the frequency range and shed a new light on the role of T-invariance at finite frequency. We will point out a danger of applying a naive fast/slow mode separation within the effective field theory. We will stress the importance of the global and gauge invariance in evaluating $\sigma(\omega)$.

\section{Dynamical Conductivity}

To be concrete, we focus ourselves on the (local) ac conductivity obtained from isolated noninteracting quantum dots with diffusive dynamics. According to the linear response theory, ac conductivity $\sigma(\omega)$ at $T=0$ is expressed as

$$
\operatorname{Re} \sigma(\omega)=\frac{\pi}{V}\left\langle\operatorname{Tr}\left[\delta(\varepsilon-H) \widehat{v}_{x} \delta(\varepsilon+\omega-H) \widehat{v}_{x}\right]\right\rangle
$$

Here the Hamiltonian $H$ contains a random or irregular potential $V(x)$, taking (we adopt $e=\hbar=1$ )

$$
H=\frac{(\widehat{\mathbf{p}}-\mathbf{A})^{2}}{2 m}+V(\mathbf{r})
$$

Instead of unjustifiable statistical hypothesis, the effective field theory assumes the ensemble average over random potential $V(x)$, denoted by $\langle\cdots\rangle$. The energy $\varepsilon$ is set to the Fermi energy; the velocity operator $\widehat{v}_{x}$ is defined as $\widehat{v}_{x}=i[H, \widehat{x}]$. We take account of Magnetic field $\mathbf{B}=\operatorname{rot} \mathbf{A}$ as a crossover between two universality classes: the unitary class with magnetic field $(\beta=2)$, or the orthogonal with time-reversal invariance $(\beta=1)$. In this paper, we neglect the level broadening, but the effect can be readily studied by introducing a complex frequency $\omega \mapsto \omega+i \gamma$. Without the level broadening, $\sigma(\omega \rightarrow+0)$ must vanish except for the Drude weight. 
We are now concerned with quantum correction of ac conductivity:

$$
\frac{\sigma(\omega)}{\sigma_{\mathrm{cl}}(\omega)}-1
$$

The (semi)-classical conductivity $\sigma_{\mathrm{cl}}$ accounts for the contribution evaluated by decoupling the average of (2.1) into each $\delta$-function:

$$
\sigma_{\mathrm{cl}}(\omega)=\pi v^{2} \int \frac{d \mathbf{r}_{1} d \mathbf{r}_{2}}{V}\left|\left\langle\mathbf{r}_{1}\left|\widehat{v}_{x}\right| \mathbf{r}_{2}\right\rangle\right|^{2} f_{\varepsilon}\left(\mathbf{r}_{1}, \mathbf{r}_{2}\right) f_{\varepsilon+\omega}\left(\mathbf{r}_{2}, \mathbf{r}_{1}\right)
$$

where $v$ is the average density of state (DOS), and the Friedel correlation $f$ describes the universal spatial correlation of the wavefunction:

$$
f_{\varepsilon}\left(\mathbf{r}_{1}, \mathbf{r}_{2}\right)=v^{-1}\left\langle\sum_{i} \psi_{i}\left(\mathbf{r}_{1}\right) \psi_{i}^{*}\left(\mathbf{r}_{2}\right) \delta\left(\varepsilon-\varepsilon_{i}\right)\right\rangle
$$

The function $f_{\varepsilon}\left(\mathbf{r}_{1}, \mathbf{r}_{2}\right)$ is directly connected with the semiclassical phase-space density through the Wigner representation. For a diffusive electron, $\sigma_{\mathrm{cl}}$ coincides with the Drude conductivity $\sigma_{0}$ for $\omega \gtrsim D / L^{2}$. However, it becomes proportional to $\omega^{2}$ for $\omega \lesssim D / L^{2}$, because $\left|\left\langle\mathbf{r}_{1}\left|\widehat{v}_{x}\right| \mathbf{r}_{2}\right\rangle\right|^{2} \propto \omega^{2}\left\langle|x|^{2}\right\rangle$ and the oscillator strength $\left\langle|x|^{2}\right\rangle$ of isolated quantum dots is finite $[1,5,6]$.

To evaluate a quantum correction equation (2.3) for $\omega \gg \Delta$, one can employ a standard diagrammatical perturbation theory. In the time-reversal invariant system $(\beta=1)$, a leading contribution comes from the Cooperon channel:

$$
\frac{\sigma_{\mathrm{per}}(\omega)}{\sigma_{0}}-1=-\frac{\Delta}{\pi} \sum_{q} \frac{1}{D q^{2}-i \omega}
$$

The result shows a positive magnetoconductance because the Cooperon is vulnerable to the time-reversal breaking,

$$
\sigma_{\text {per }}(\omega, B)-\sigma_{\text {per }}(\omega, B=0)>0
$$

When $\omega$ decreases up to the order of the mean level spacing $\Delta$, level repulsion should be visible. We no longer utilize the diagrammatic theory; we need a nonperturbative treatment, that is, the supermatrix NL- $\sigma$ model, or RMT with statistical hypothesis. Earlier studies along this line started with examining the spatial dependence of the two-particle correlator (see (3.7)); they were successful in understanding the validity of GE result by the literal expansion of $1 / \mathrm{g}$. We stress that the analysis so far is incomplete to cover the entire range of $\omega$. When $\omega$ exceeds $\Delta$, we should see $\sigma(\omega)$ gradually changing itself into the perturbative form (2.6); yet such a behavior cannot be captured by a finite order of $1 / \mathrm{g}$ expansions, since the diffusion form can appear only through resummation of all the order of $1 / g$ expansion. 
Restoring the correct perturbation result for $\omega \gg \Delta$ has another fundamental significance. If $\sigma(\omega)$ were to be proportional to the DOS correlator, it would mean $\sigma(\omega) \propto$ $\ln ^{2} \omega$ in $2 \mathrm{D}$, but such behavior is incompatible with the RG treatment of conductivity. The role of T-invariance has to be reconsidered dynamically depending on the frequency range (see also experiments $[12,13])$.

\section{Summary of Results and Discussion}

To resolve and clarify the role of T-invariance at finite frequency, we evaluate a quantum correction of the linear ac conductivity by resorting to the supermatrix NL- $\sigma$ model, which provides a nonperturbative description of (low-energy) diffusion modes. A subtlety appears in considering how to reproduce $\sigma_{\mathrm{cl}}$. Since $\sigma_{\mathrm{cl}}$ is a high-energy contribution, it should be implemented as a saddle point solution. Indeed, to reproduce (2.4), we need a nonlocal saddle point solution $Q\left(\mathbf{r}_{1}, \mathbf{r}_{2}\right) \propto f\left(\mathbf{r}_{1}, \mathbf{r}_{2}\right)$. A further spatial/spectral coarse-graining restores a local diffusive action as the effective field theory defined on the symmetric space as a result of gauging out the massive degrees of freedom.

By implementing the above modification, the effective action with diffusive dynamics becomes (see our conventions in Appendix A)

$$
F[Q]=-\frac{\pi}{c} \int \operatorname{STr}\left[\sigma_{0}(\nabla Q)^{2}+2 i v \omega Q \Lambda\right] d \mathbf{r}
$$

\subsection{Dynamical Conductivity by Invariant Correlators}

By devoting all the derivation to the next section and appendices, we write our result as the following exact, nonperturbative expression of the quantum correction:

$$
\frac{\sigma(\omega)}{\sigma_{\mathrm{cl}}(\omega)}-1=k(\mathbf{0})+n_{C}(\mathbf{0})-\frac{16 \pi \sigma_{\mathrm{cl}}}{c V} \int \boldsymbol{\Phi}_{x x}(\mathbf{r}) d \mathbf{r}
$$

The quantum correction is found to consist of three terms: the DOS fluctuation $k(\mathbf{0})$, the "Cooperon density" fluctuation $n_{C}(\mathbf{0})$ present only for time-reversal invariant system, and $\Phi(\mathbf{r})$ from the (global) current correlator of the supermatrix NL- $\sigma$ model. The average $\langle\cdots\rangle$ now means the integration over $Q$ with the weight $e^{-F[Q]}$. Each term in (3.2) is expressed as an invariant correlator of the NL- $\sigma$ model:

$$
\begin{gathered}
k\left(\mathbf{r}-\mathbf{r}^{\prime}\right)=-\frac{1}{c^{2}}\left\langle\operatorname{STr}[k Q(\mathbf{r})] \operatorname{STr}\left[k Q\left(\mathbf{r}^{\prime}\right)\right]\right\rangle, \\
n_{C}\left(\mathbf{r}-\mathbf{r}^{\prime}\right)=-\frac{\delta_{\beta, 1}}{c^{2}}\left\langle\operatorname{STr}\left[k Q(\mathbf{r}) k \bar{Q}\left(\mathbf{r}^{\prime}\right)\right]\right\rangle, \\
\boldsymbol{\Phi}_{\mu v}\left(\mathbf{r}-\mathbf{r}^{\prime}\right)=\frac{1+\delta_{\beta, 1}}{c^{2}}\left\langle\operatorname{STr}\left[k \partial_{\mu}(\mathbf{r}) k \partial_{\nu}\left(\mathbf{r}^{\prime}\right)\right]\right\rangle,
\end{gathered}
$$

where $\bar{Q}$ is defined in Appendix A; the Noether current is $\partial_{\mu}=(1 / 2) Q \partial_{\mu} Q$. The above are written for $\beta=1,2$; the symplectic class $\beta=4$ can be incorporated by reversing the sign in 
front of $n_{C}(\mathbf{0})$. Note that a quantum correction is controlled by a coarse-grained dynamics, so that $\sigma_{0}$ appears in the effective field theory. This is the main result of the paper.

Equation (3.2) is exact; it has the same validity as the supermatrix NL- $\sigma$ model itself, describing all the perturbative/nonperturbative effects fully, since it is derived within the effective theory through rewriting (2.1) without any further approximation. To examine a concrete dependence of $\sigma(\omega)$, we can readily utilize various well-controlled approximations available to the $Q$-matrix theory, either for $\omega \gtrsim \Delta$ or for $\omega \lesssim \Delta$.

Though expressions similar to (3.2) can be found in literatures, they miss incorporating some ingredients due to nonperturbative and/or massive contributions. The formula substituting constant $\sigma_{0}$ for $\sigma_{\mathrm{cl}}$ has been found before [14]. However such an expression implies a low-frequency behavior $\operatorname{Re} \sigma(\omega) \propto \omega^{\beta}$ (for $\beta=1,2$ for the orthogonal/unitary class), not the form $\omega^{2+\beta}$ suggested in $[1,5,6]$. The missing exponent will be explained by a proper treatment of massive contribution $\sigma_{\mathrm{cl}} \alpha \omega^{2}$ in the present paper. Indeed, the appearance of frequency-dependent $\sigma_{\mathrm{cl}}(\omega)$ rather than $\sigma_{0}$ is mandatory as a consequence of the correct (gauge) invariance of the effective theory. In addition, there seems some interesting discrepancy with replica methods in the nonperturbative region, as will be discussed later. Both of these massive and nonperturbative contributions become important and dominant to the dynamical conductivity in small metallic grains.

\subsection{Zero-Dimensional Limit $\omega \ll \Delta$}

When we are interested in $\omega \ll \Delta$, we may use the 0D approximation of $Q$-matrix. In the lowest-order approximation, we can neglect the third term and only the first and the second term contribute. By seeing the level correlator is given by $R(s)=1+V^{-1} \operatorname{Re} \int k(\mathbf{r}) d \mathbf{r}$, ac conductivity becomes

$$
\frac{\sigma(\omega)}{\sigma_{\mathrm{cl}}} \approx \mathcal{R}_{\mathrm{wd}}\left(\frac{\omega}{\Delta}\right)+\frac{\Delta}{-i \pi \omega} \delta_{\beta, 1}
$$

where $\mathcal{R}_{\mathrm{wd}}(s)$ is a complexified function of the Wigner-Dyson level correlator $R_{\mathrm{wd}}(s)$, that is, $\operatorname{Re} \mathcal{R}_{\mathrm{wd}}(s)=R_{\mathrm{wd}}(s)$. This reproduces the GE result, corrected by the second term from $n_{C}$ in the time-reversal invariant system. The second term has exactly the same origin of enhancement in the inverse participation ratio in a time-reversal invariant system and of the positive magnetopolarizability $[10,11]$.

Higher corrections of $1 / g^{n}(n=1,2)$ were obtained by the literal expansion of $1 / g$ around $0 \mathrm{D}$ limit $[8,9]$. As for $n_{C}$, the result is consistent with its perturbative form in $\omega \gg \Delta$ :

$$
n_{\mathrm{C}}(0 ; \omega \gg \Delta) \approx \frac{\Delta}{\pi} \sum_{q} \frac{1}{D q^{2}-i \omega}
$$

One may naively think that this may lead to the conventional weak localization effect, but beware of the opposite sign! Rather, the local terms $k(0)$ and $n_{C}(\mathbf{0})$ of (3.2) serve as negative magnetoconductance. The effect of time-reversal invariance/breaking is delicate. For the orthogonal case, the third term, which also becomes a diffusive form with twice as large as $n_{C}(0)$, cancels out $n_{C}$ and reproduces the standard diagrammatical result (2.6) with $\sigma_{\mathrm{cl}}$ instead of $\sigma_{0}$. In contrast, the unitary case is even more subtle with the $1 / g$-correction absent. 
The term $k(\mathbf{0})$ contributes as the same order as the current correlator. Combining these, we have a perturbative result:

$$
\frac{\sigma_{\mathrm{per}}(\omega)}{\sigma_{\mathrm{cl}}}-1=\left(2-\frac{4}{d}\right)\left(\frac{\Delta}{\pi} \sum_{q} \frac{1}{D q^{2}-i \omega}\right)^{2} .
$$

In $d \rightarrow 2$, the correction needs a careful treatment; the dimensional regularization provides a familiar logarithmic dependence $\ln \omega$ at the order of $O\left(1 / g^{2}\right)$, which conforms to the RG analysis.

\subsection{Connection with the Decomposition Formula: Global and Gauge Symmetries}

To understand further the nature of (3.2) as well as elucidating subtleties in earlier calculations by NL- $\sigma$ approach, we compare the form with the "decomposition formula" [15], which underlies the previous evaluations of dynamical responses in the nonperturbative region [8-10]. A large $g$ allows us to decompose spatial correlation of the two-particle Green function into the fast and slow spatial dependence, whose contribution consists of the three channels: $k, n_{C}$ defined as before, and the Diffuson channel $n(\mathbf{r})=-c^{-2}\langle\operatorname{STr}[k Q(\mathbf{r}) k Q(\mathbf{0})]\rangle$ [15]:

$$
\begin{aligned}
& \mathcal{v}^{-2}\left\langle\left\langle\mathbf{r}_{1}|\delta(\varepsilon+\omega-H)| \mathbf{r}_{2}\right\rangle\left\langle\mathbf{r}_{2}|\delta(\varepsilon-H)| \mathbf{r}_{1}\right\rangle\right\rangle \\
& \quad=\operatorname{Re}\left[\left|f\left(\mathbf{r}_{12}\right)\right|^{2}\left(1+k(\mathbf{0})+n_{C}(\mathbf{0})\right)+n\left(\mathbf{r}_{12}\right)\right] .
\end{aligned}
$$

One might expect to obtain (3.2) by putting the above in the coordinate representation of (2.1). Indeed, by applying the velocity operators, we see that the terms $k(0)$ and $n_{C}(0)$ agree exactly in two expressions. However, discrepancy exists in the third term. It arises from the way one uses the effective field theory, either after or before the operator (matrix element) insertion. Within the effective field theory, localization effect is encoded as interaction between diffusion modes arising from the nonlinear constraint $Q^{2}=1$. Not only $Q$ is "gaugeinvariant" but also the NL- $\sigma$ model has a global symmetry, which leads to the (nonlinear) diffusion equation $2 D \nabla(Q \nabla Q)=-i \omega[\Lambda, Q]$. Both of the symmetries must be conserved. One can immediately see the danger in inserting the operator $\nabla$ (in $\widehat{v}$ ) on $n(\mathbf{r})$ because the kinetic equation with nonlinear constraint induces not only a linear term of $Q$ but also new composite fields. To get a meaningful result, these "higher-order" contributions must be treated not to violate both of the global and the gauge symmetries. The present argument also highlights the importance of formulating the problem in terms of invariant correlators defined in (3.3). Otherwise one could not convince oneself whether the invariance is manifested or not. 


\subsection{Incorporating Approximation}

A reliable estimate of ac conductivity in a finite volume system is obtained by taking account both of the zero-mode integration and perturbative contributions around it [16]. After some straightforward but elaborate calculation for (3.2), we obtain the result for the unitary case:

$$
\begin{aligned}
\frac{\sigma(\omega)}{\sigma_{\mathrm{cl}}}= & \mathcal{R}_{\mathrm{wd}}(s)\left[1+\frac{2(d-2)}{d} \Pi_{1}^{2}(\omega)-\frac{4(d+3)}{d} \Pi_{2}(\omega)\right] \\
& -\frac{2}{i \pi s} \frac{d-2}{d} \Pi_{1}(\omega)+\left[\frac{4(d-2)}{d} s \mathcal{R}_{\mathrm{wd}}^{\prime}(s)+s^{2} \mathcal{R}_{\mathrm{wd}}^{\prime \prime}(s)\right] \Pi_{2}(\omega),
\end{aligned}
$$

where $s=\omega / \Delta$, and $\Pi_{k}(\omega)$ denotes a contribution from the diffusion propagator:

$$
\Pi_{k}(\omega)=\frac{\Delta^{k}}{\pi^{k}} \sum_{q} \frac{1}{\left(D q^{2}-i \omega\right)^{k}}
$$

A similar but more involved expression can be obtained for the orthogonal case. It shows that except for a zero-mode contribution, a dominant (anomalous) term coincides with a perturbative behavior; nonperturbative effect is confined approximately in the factor $\mathcal{R}_{\mathrm{wd}}(s)$. The above observation implies that as a practical incorporating approximation, we can use the following form:

$$
\frac{\sigma(\omega)}{\sigma_{\mathrm{cl}}} \approx \mathcal{R}_{\mathrm{wd}}\left(\frac{\omega}{\Delta}\right) \frac{\sigma_{\mathrm{per}}(\omega)}{\sigma_{0}}
$$

for all universality classes, except for the zero-mode contribution of $n_{C}$. The above form is anticipated when one attempts to make both the GE behavior for small $\omega$ and the RG behavior for large $\omega$ compatible. We have provided a solid justification microscopically through the NL- $\sigma$ model.

\section{Invariant Formulation}

\section{1. $\sigma(\omega)$ in the NL- $\sigma$ Model}

We now outline how the expression (3.2) is derived. Following the approach [17-19], we first rewrite the linear response $\sigma(\omega)$ directly within the framework of NL- $\sigma$ model by introducing the external (background) gauge field $\mathscr{A}$. Being expanded up to $O\left(\mathscr{A}^{2}\right)$ in the exponent, we define the observable $\sigma(\omega)$, which is known to be equivalent to the linear response theory. The effect of the external field $\mathcal{A}$ is incorporated into NL- $\sigma$ model by $Q \mapsto Q_{g}=g Q g^{-1}$ where 
$\mathcal{A}=g^{-1} \nabla g$. The symmetry of the effective theory requires the generating functional $Z[\mathcal{A}]$ to take the following form for any choice of external field $A$ :

$$
\begin{aligned}
Z[\mathcal{A}]= & \int D Q \exp \left(-F\left[g Q g^{-1}\right]\right) \\
& =\exp \frac{\pi}{c} \int \operatorname{STr}\left[\sigma(\omega)\left(\nabla Q_{g}\right)^{2}+2 i v \omega Q_{g} \Lambda\right] d \mathbf{r} .
\end{aligned}
$$

Here we define the observable $\sigma(\omega)$ on the right-hand side within the effective field theory. Comparing between both sides up to $O\left(\mathcal{A}^{2}\right)$, we find $\sigma(\omega)$ as

$$
\frac{\sigma(\omega)}{\sigma_{\mathrm{cl}}} \mathrm{S} \operatorname{Tr}[\mathcal{A}, \Lambda]^{2}=\left\langle\mathrm{S} \operatorname{Tr}[\mathcal{A}, Q(\mathbf{r})]^{2}\right\rangle+\frac{2 \pi \sigma_{\mathrm{cl}}}{c V}\left\langle\left(\int \operatorname{STr} \nabla Q(\mathbf{r})[\mathcal{A}, Q(\mathbf{r})] d \mathbf{r}\right)^{2}\right\rangle
$$

\subsection{Symmetrization into Invariant Correlators}

Though (4.2) above may be useful to evaluate $\sigma(\omega)$, an explicit dependence on the choice of $\mathcal{A}$ conceals the global and gauge symmetry of the theory, inconvenient for an actual evaluation. So we uncover the full symmetry by symmetrizing $\mathcal{A}$. Following the idea in $[18,19]$, we recast the formula in terms of invariant correlators. In replica theories, such a symmetrization procedure is readily completed by using the Fierz identity. In the present case, however, symmetrizing $\mathbb{A}$ over the relevant symmetry, that is, the superalgebra $\mathfrak{g l}(2 \mid 2)$ for the unitary case $(\mathfrak{o s p}(4 \mid 4)$ for the orthogonal) requires more careful treatment; the source field $\mathcal{A}$ belongs to the Lie algebra with Grassmann structure rather than the Lie superalgebra itself [20]; we need to modify the relevant Fierz identities accordingly. As will be shown in Appendix B, a cautious symmetrization over the basis $\mathcal{A}=X_{i} \in \mathfrak{g l}(m \mid n)$ leads to the following rules for $\mathfrak{g l}(m \mid n)$ :

$$
\begin{aligned}
& \mathrm{S} \operatorname{Tr}\left[X_{i} A\right] \mathrm{S} \operatorname{Tr}\left[X_{i} B\right] \longrightarrow \mathrm{S} \operatorname{Tr}[k A k B], \\
& \mathrm{S} \operatorname{Tr}\left[X_{i} A X_{i} B\right] \longrightarrow \mathrm{S} \operatorname{Tr}[k A] \mathrm{S} \operatorname{Tr}[k B],
\end{aligned}
$$

where $A$ and $B$ are arbitrary supermatrices. For the time-reversal invariant system, $\mathcal{A}$ has to be restricted to the orthosymplectic algebra $\mathfrak{o s p}(4 \mid 4)$, which is the time-reversal invariant subspace of $\mathfrak{g l}(4 \mid 4)$. A resulting symmetrization rule for $\mathfrak{o s p}$ is found to be (Appendix B)

$$
\begin{gathered}
2 \mathrm{~S} \operatorname{Tr}\left[X_{i} A\right] \mathrm{S} \operatorname{Tr}\left[X_{i} B\right] \longrightarrow \mathrm{S} \operatorname{Tr}[k A k(B+\bar{B})], \\
2 \mathrm{~S} \operatorname{Tr}\left[X_{i} A X_{i} B\right] \longrightarrow \mathrm{S} \operatorname{Tr}[k A] \mathrm{S} \operatorname{Tr}[k B]-\mathrm{S} \operatorname{Tr}\left[k A C B^{t} C\right] .
\end{gathered}
$$

By using the above symmetrization, (4.2) is found to be equal to (3.2). 


\subsection{Comparison with Replica Formulations}

Quite interestingly, a striking discrepancy between supermatrix and replica formulations emerges upon symmetrization. In the study of RG treatment of the conductivity, corresponding formulae (for the ratio with $\sigma_{0}$ ) have been derived long before in replica methods. While an unsymmetrized formula corresponding to (4.2) looks almost identical [17], a symmetrized formula in the replica method $[18,19]$ comprises only the third term, $\Phi_{x x}$ of $(3.2)$ for all three universality classes. This is so because, instead of (4.3) and (4.4), symmetrization rules for replica theories are (See Appendix B)

$$
\begin{aligned}
& \operatorname{Tr}\left[X_{i} A\right] \operatorname{Tr}\left[X_{i} B\right] \longrightarrow \operatorname{Tr}[A B], \\
& \operatorname{Tr}\left[X_{i} A X_{i} B\right] \longrightarrow \operatorname{Tr}[A] \operatorname{Tr}[B]
\end{aligned}
$$

Accordingly, no local terms survive after symmetrization.

In the perturbative treatment, such a difference does not show up. Both the symmetrized and the unsymmetrized replica/supermatrix methods produce exactly the same perturbative RG results for all the three universality classes [17-19]. On the other hand, this is not true for nonperturbative contributions. Though some successful attempts exist to try to obtain nonperturbative Wigner-Dyson correlations [21-23], it is unclear whether the replica method will be able to account for a full nonperturbative effect such as (3.8) or (3.10), to the author's knowledge. The supermatrix formulation clearly has a better handle to work on both perturbative and nonperturbative contributions.

\section{Conclusion}

In conclusion, we have examined the dynamical conductivity $\sigma(\omega)$ in small metallic grains through invariant correlators of the supermatrix NL- $\sigma$ model. The present formulation allows us to understand that T-invariance can either enhance or reduce $\sigma(\omega)$, depending on the frequency range. We have provide a unified point of view in understanding various perturbative/nonperturbative and massive/massless contributions. Our expression (3.2) is quite generic, derived from the symmetry of the effective field theory. Yet it is of much practical use in evaluating dynamical responses. A striking feature of the formula is the presence of local terms, $k(0)$ and $n_{C}(0)$. We believe that the formula will gives a reliable way to account for various interesting nonperturbative effects such as (multi)fractality or "prelocalized states" in the conductivity. Our derivation is algebraic, so that the result can be extended to other universality classes described by supermatrix NL- $\sigma$ models [24].

\section{Appendices}

\section{A. Notational Conventions}

We introduce $c \times c$ supermatrices and define the even/odd grading $|i|=0$ (1) for a bosonic (fermionic) component. We define the superparity(=grading) matrix $k_{i j}=(-1)^{|i|} \delta_{i j}$, and the supertrace by $\operatorname{STr}[M]=\operatorname{Tr}[k M]$. The general linear superalgebra $\mathfrak{g l}(m \mid n)$ is a generic supermatrix with the dimension of $(m+n) \times(m+n)$. The orthosymplectic algebra osp $(2 m \mid 2 n)$ is a time-reversal invariant subspace of $\mathfrak{g l}(2 m \mid 2 n)$. We define the time-reversal matrix $C$ 
to conform to the standard definition of $\mathfrak{o s p}(n \mid n)$ in literatures by introducing the timereversal operation $\bar{X} \equiv-C^{-1} X^{t} C$. Note $C^{2}= \pm k$ for the orthogonal/symplectic class. The supermatrix field $Q$ is defined as the adjoint representation of $\mathfrak{g}$, isomorphic to the symmetric space $G /(K \times K)$, where $\mathfrak{g}=\mathfrak{g l}(2 \mid 2), G=G L(2 \mid 2)$ and $K=G L(1 \mid 1)$ for the unitary universal class; $\mathfrak{g}=\mathfrak{o s p}(2 \mid 2), G=\operatorname{OSp}(2 \mid 2)$ and $K=\operatorname{OSp}(1 \mid 1)$ for the orthogonal/symplectic universality class.

\section{B. Symmetrization Procedures}

\section{B.1. Symmetrization of $\mathfrak{g l}(n)$}

For conventional Lie algebras, the symmetrization is completed simply by the Fierz identity (the Casimir relation). For actual calculations, we find it advantageous to work on the standard basis $\left(e_{a b}\right)_{i j}:=\delta_{a i} \delta_{b j}$ for $\mathfrak{g l}(n)$ rather than any particular choice of orthogonal basis $X_{i}$. The Fierz identity for $\mathfrak{g l}(n)$ on the standard basis can be checked by a direct calculation as

$$
\sum_{a, b, c, d} g^{a b, c d}\left(e_{a b}\right)_{i j}\left(e_{c d}\right)_{k l}=\delta_{i l} \delta_{j k}
$$

where $g_{a b, c d}:=\operatorname{Tr}\left[e_{a b} e_{c d}\right]=\delta_{a d} \delta_{b c}$ is a metric and $g^{a b, c d}$ is the inverse of it. Note that $g_{a b, c d}$ is not the Cartan metric defined by the Killing form, but the one associated with the invariant bilinear form $B(X, Y)=\operatorname{Tr}[X Y]$. In replica formulations, we can utilize the above Fierz identity for symmetrizing as

$$
\begin{aligned}
& \sum_{a, b, c, d} g^{a b, c d} \operatorname{Tr}\left[e_{a b} A\right] \operatorname{Tr}\left[e_{c d} B\right]=\operatorname{Tr}[A B], \\
& \sum_{a, b, c, d} g^{a b, c d} \operatorname{Tr}\left[e_{a b} A e_{c d} B\right]=\operatorname{Tr}[A] \operatorname{Tr}[B] .
\end{aligned}
$$

\section{B.2. Symmetrization of $\mathfrak{g l}(m \mid n)$}

We next extend the Fierz identity above to Lie superalgebras $\mathfrak{g l}(m \mid n)$. We can achieve it by substituting the invariant bilinear form $B(X, Y)=S \operatorname{Tr}[X Y]$ and taking account of anticommuting properties correctly. In doing so, we need to bookkeep a component $i$ by the even/odd grading $|i|$. For a standard basis $\left(e_{a b}\right)_{i j}=\delta_{a i} \delta_{b j}$ of $\mathfrak{g l}(m \mid n)$, an explicit calculation leads to

$$
\sum_{a, b, c, d} g^{a b, c d}\left(e_{a b}\right)_{i j}\left(e_{c d}\right)_{k l}=(-1)^{|j|} \delta_{i l} \delta_{j k}
$$

where the (nonsymmetric) metric is defined by

$$
\begin{gathered}
g_{a b, c d}=\mathrm{S} \operatorname{Sr}\left[e_{a b} e_{c d}\right]=(-1)^{|a|} \delta_{a d} \delta_{b c}, \\
g^{a b, c d}=\left(g_{a b, c d}\right)^{-1}=(-1)^{|b|} \delta_{a d} \delta_{b c} .
\end{gathered}
$$




\section{B.3. Symmetrization of Lie Algebras with the Grassmann Structure}

Finally we investigate the symmetrization for Lie algebras with the Grassmann structure, which is needed for (4.3) and (4.4). Instead of using a bosonic/fermionic $e_{a b}$ satisfying the Lie superalgebra, we use a new base $E_{a b}=\lambda_{a b} e_{a b}$ including a corresponding bosonic/fermionic Grassmann parameter $\lambda_{a b}$. Note that $E_{\mathrm{a} b}$ satisfies the Lie algebra with a usual bracket, and the field $\mathcal{A}=X_{i}$ in (4.3) belongs to the algebra generated by $E_{a b}$ rather than the superalgebra itself.

Now let us consider the symmetrization

$$
\mathrm{S} \operatorname{Tr}\left[E_{a b} A\right] \mathrm{S} \operatorname{Tr}\left[E_{c d} B\right],
$$

which is a counterpart of (4.3) in the standard basis. We see that terms with the grading $\left|E_{a b}\right|\left|E_{c d}\right|=1$ have an antisymmetry in exchanging $E_{a b}$ and $E_{c d}$, so that a correct symmetrization is

$$
\sum_{a, b, c, d}(-1)^{\left|E_{a b}\right|\left|E_{c d}\right|} g^{a b, c d} \operatorname{STr}\left[E_{a b} A\right] \mathrm{S} \operatorname{Tr}\left[E_{c d} B\right]
$$

A straightforward calculation using (B.3) leads to

$$
\begin{aligned}
& \sum_{a, b, c, d}(-1)^{\left|E_{a b}\right|\left|E_{c d}\right|} g^{a b, c d} \operatorname{STr}\left[E_{a b} A\right] \mathrm{S} \operatorname{Tr}\left[E_{c d} B\right] \\
& =\sum_{i, j}(-1)^{|j|} \lambda_{i j} \lambda_{j i}(A k)_{j i}(B k)_{i j}=\mathrm{S} \operatorname{Sr}[A k B k],
\end{aligned}
$$

where $\lambda_{i j} \lambda_{j i}=1$ in the last equality.

Equation (4.4) can be derived similarly by considering

$$
\sum_{a, b, c, d}(-1)^{\left|E_{a b}\right|\left|E_{c d}\right|} g^{a b, c d} \operatorname{STr}\left[E_{a b} A E_{c d} B\right]
$$

\section{Orthosymplectic Algebras}

Since the orthosymplectic algebras $\mathfrak{o s p}(2 m \mid 2 n)$ is a T-invariant subspace of $\mathfrak{g l}(2 m \mid 2 n)$, the only modification needed is to restrict all the basis onto the invariant subspace satisfying $X_{i}=\bar{X}_{i}$. We can construct those T-invariant bases $\epsilon_{a b}$ from $e_{a b} \in \mathfrak{g l}(2 m \mid 2 n)$ as

$$
\begin{gathered}
\epsilon_{a b}=\frac{1}{2}\left(e_{a b}+\overline{e_{a b}}\right), \\
\overline{e_{a b}}=-(-1)^{|a||b|} k C^{-1} e_{b a} C .
\end{gathered}
$$

We can follow the same steps for $\mathfrak{o s p}(2 m \mid 2 n)$ as for the $\mathfrak{g l}(m \mid n)$, obtaining the symmetrization. 


\section{Acknowledgment}

The author appreciates partial support by Grant-in-Aid for Scientific Research from the Ministry of Education, Culture, Sports, Science and Technology of Japan (Grant no. 22540324), in completing the present work.

\section{References}

[1] L. P. Gor'kov and G. M. Eliashberg, "Minute metallic particles in an electromagnetic field," Soviet Physics-JETP, vol. 21, p. 940, 1965.

[2] M. L. Mehta, Random Matrices, Academic Press, Boston, Mass, USA, 2nd edition, 1991.

[3] S. Strässler, M. J. Rice, and P. Wyder, "Comment on Gorkov and Eliashberg's result for the polarizability of a minute metallic particle," Physical Review B, vol. 6, no. 7, pp. 2575-2577, 1972.

[4] M. J. Rice, W. R. Schneider, and S. Strässler, "Electronic polarizabilities of very small metallic particles and thin films," Physical Review B, vol. 8, no. 2, pp. 474-482, 1973.

[5] U. Sivan and Y. Imry, "Energy-level correlation function and ac conductivity of a finite disordered system," Physical Review B, vol. 35, no. 12, pp. 6074-6083, 1987.

[6] K. Frahm, B. Mühlschlegel, and R. Németh, "Size dependent ac conductivity of small metal particles," Zeitschrift für Physik B, vol. 78, no. 1, pp. 91-97, 1990.

[7] K. B. Efetov, "Supersymmetry and theory of disordered metals," Advances in Physics, vol. 32, no. 1, pp. 53-127, 1983.

[8] Y. M. Blanter and A. D. Mirlin, "Gor'kov and Eliashberg linear-response theory: rigorous derivation and limits of applicability," Physical Review B, vol. 53, no. 19, pp. 12601-12604, 1996.

[9] A. D. Mirlin, "Statistics of energy levels and eigenfunctions in disordered systems," Physics Reports, vol. 326, no. 5-6, pp. 259-382, 2000.

[10] K. B. Efetov, "Polarizability of small metal particles: “Weak localization" effects," Physical Review Letters, vol. 76, no. 11, pp. 1908-1911, 1996.

[11] R. Deblock, Y. Noat, H. Bouchiat, B. Reulet, and D. Mailly, "Measurements of flux-dependent screening in aharonov-bohm rings," Physical Review Letters, vol. 84, no. 23, pp. 5379-5382, 2000.

[12] B. Reulet, M. Ramin, H. Bouchiat, and D. Mailly, "Dynamic response of isolated aharonov-bohm rings coupled to an electromagnetic resonator," Physical Review Letters, vol. 75, no. 1, pp. 124-127, 1995.

[13] Y. Noat, H. Bouchiat, B. Reulet, and D. Mailly, "Sign reversals of ac magnetoconductance in isolated quantum dots," Physical Review Letters, vol. 80, no. 22, pp. 4955-4958, 1998.

[14] K. B. Efetov and S. Iida, "Dynamics and thermodynamics of a system of mesoscopic metallic rings," Physical Review B, vol. 47, no. 23, pp. 15794-15808, 1993.

[15] N. Taniguchi, B. D. Simons, and B. L. Altshuler, "Spectral correlation and response functions in quantum dots," Physical Review B, vol. 53, no. 12, pp. R7618-R7621, 1996.

[16] V. E. Kravtsov and A. D. Mirlin, "Levels statistics in a metallic sample: corrections to the WignerDyson distribution," JETP Letters, vol. 60, p. 656, 1994.

[17] V. E. Kravtsov and I. V. Lerner, "Direct calculation of the conductance of disordered media using $\sigma$-model approach," Soviet Physics. Solid State, vol. 29, p. 259, 1987.

[18] A. M. M. Pruisken, "Quasiparticles in the theory of the integral quantum Hall effect (I)," Nuclear Physics B, vol. 285, pp. 719-759, 1987.

[19] A. M. M. Pruisken and Z. Wang, "Some critical properties of $G / H$ non-linear sigma models near two dimensions. I. Universal scaling law for the coupling constant," Nuclear Physics B, vol. 322, no. 3, pp. 721-737, 1989.

[20] F. A. Berezin, Introduction to Superanalysis, vol. 9 of Mathematical Physics and Applied Mathematics, D. Reidel, Dordrecht, The Netherlands, 1987.

[21] A. Kamenev and M. Mézard, "Level correlations in disordered metals: the replica $\sigma$ model," Physical Review B, vol. 60, no. 6, pp. 3944-3954, 1999.

[22] I. V. Yurkevich and I. V. Lerner, "Delocalization in an open one-dimensional chain in an imaginary vector potential," Physical Review Letters, vol. 82, no. 25, pp. 5080-5083, 1999.

[23] M. R. Zirnbauer, "Another critique of the replica trick," preprint, 1999, http://arxiv.org/abs/condmat/9903338

[24] M. R. Zirnbauer, "Riemannian symmetric superspaces and their origin in random-matrix theory," Journal of Mathematical Physics, vol. 37, no. 10, pp. 4986-5018, 1996. 


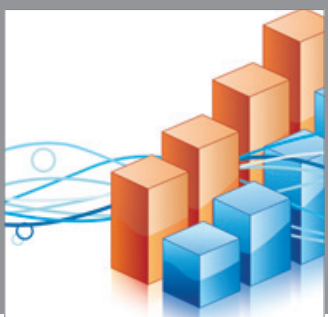

Advances in

Operations Research

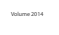

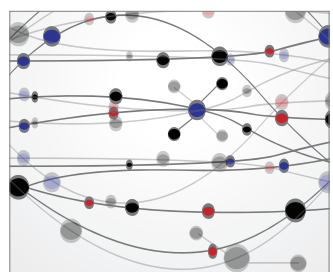

\section{The Scientific} World Journal
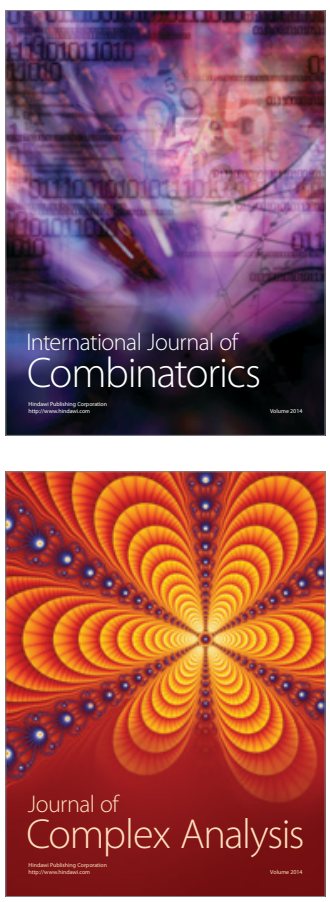

International Journal of

Mathematics and

Mathematical

Sciences
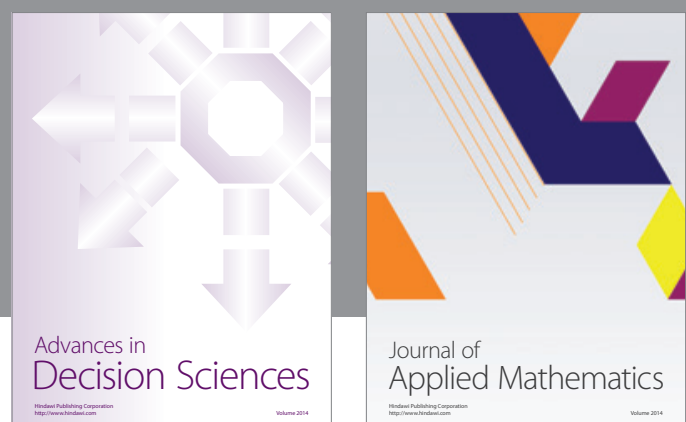

Journal of

Applied Mathematics
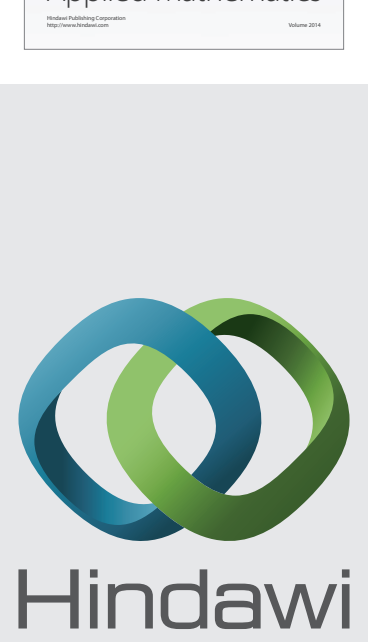

Submit your manuscripts at http://www.hindawi.com
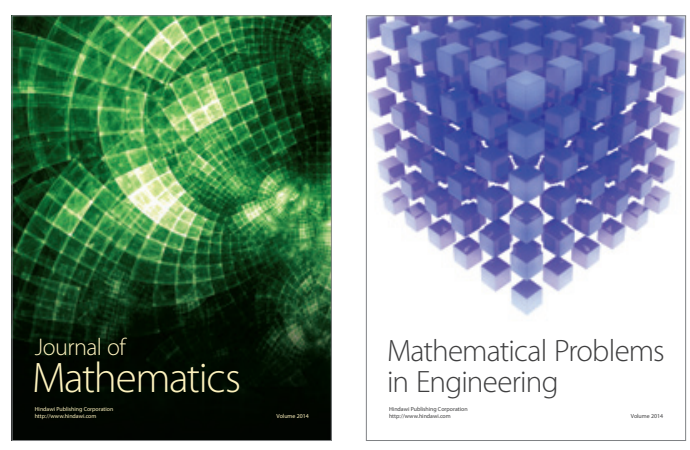

Mathematical Problems in Engineering
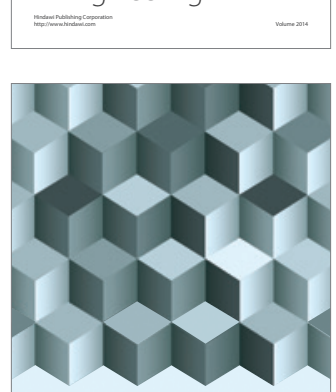

Journal of

Function Spaces
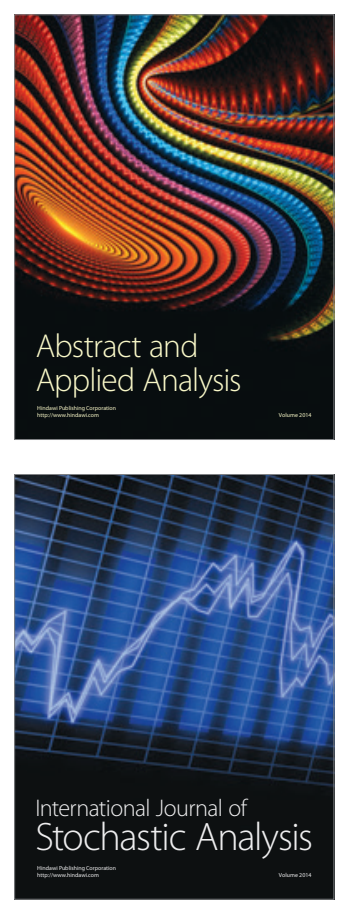

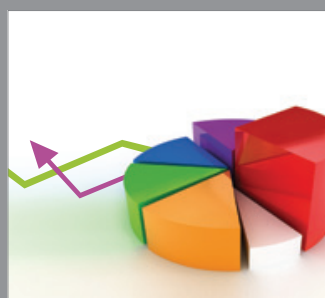

ournal of

Probability and Statistics

Promensencen
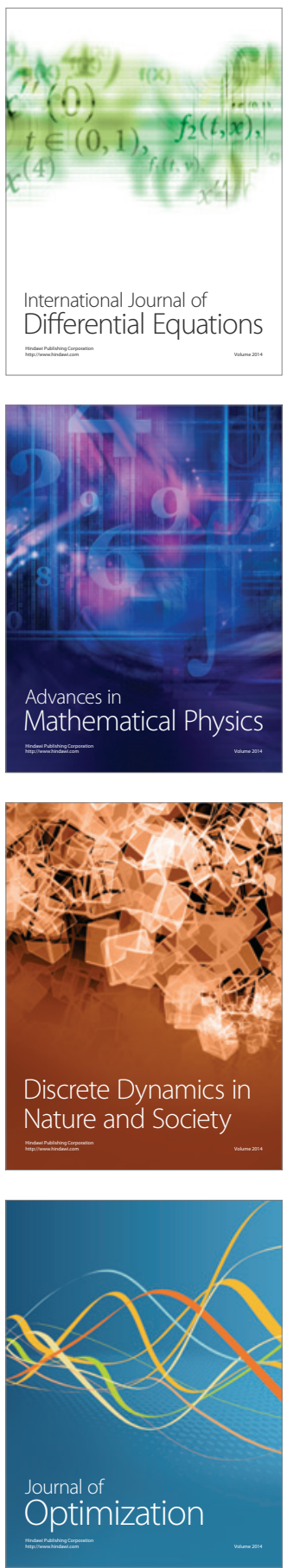